\title{
RECURRENT SURFACE HOMEOMORPHISMS
}

\author{
BORIS KOLEV AND MARIE-CHRISTINE PÉROUÈME
}

\begin{abstract}
An orientation-preserving recurrent homeomorphism of the two-sphere which is not the identity is shown to admit exactly two fixed points. A recurrent homeomorphism of a compact surface with negative Euler characteristic is periodic.
\end{abstract}

\section{INTRODUCTION}

A homeomorphism $f$ of a compact metric space $(X, d)$ is recurrent if it admits iterates arbitrarily close to the identity, i.e. if there exists a sequence $n_{k} \rightarrow+\infty$ such that

$$
d\left(f^{n_{k}}, I d\right) \rightarrow 0 \quad \text { as } \quad k \rightarrow+\infty
$$

This notion is in fact independent of the metric $d$ which defines the topology on $X$, and is invariant under topological conjugacy. The set of recurrent homeomorphisms of $X$ includes periodic homeomorphisms and more generally regular homeomorphisms $[1,8]$, i.e. homeomorphisms such that the sequence of all iterates forms an equicontinuous family:

$$
\forall \varepsilon>0, \exists \eta>0 ; \quad d(x, y)<\eta \Rightarrow d\left(f^{k}(x), f^{k}(y)\right)<\varepsilon, \quad \forall k \in \mathbb{Z} .
$$

In the case of the two-sphere $\mathbb{S}^{2}$, regular homeomorphisms have been completely classified by Kerékjártó. They are conjugate to the restriction of Euclidean isometries of the ambient 3-space [1, 5, 10, 11].

In [13], Kerékjártó asked if a non-periodic, orientation-preserving, recurrent homeomorphism of the sphere is always conjugate to an irrational rotation. The answer is now known to be false. In [7], Fokkink and Oversteegen gave an example of a periodic-point free, recurrent homeomorphism of the annulus which is not conjugate to an irrational rotation. A similar construction gives an orientation-preserving, recurrent homeomorphism of $\mathbb{S}^{2}$ with just two fixed points (and no other periodic points) that is not conjugate to an irrational rotation. In that example, the homeomorphism leaves invariant, a non-locally connected continuum $H$ with a dense orbit even if it is not minimal: the orbit closure of each point contains a fixed point. Besides the fact that recurrence does not imply regularity, this example shows that the collection of orbit closures may not even form a partition of $\mathbb{S}^{2}$. However, we will establish here the following result:

Date: August 12, 1996.

1991 Mathematics Subject Classification. 37B20, 37E30, 54H20, 57N05.

The authors express their gratitude to Alexis Marin for several remarks that helped to improve this paper. 
Theorem 1.1. A non-trivial, orientation-preserving and recurrent homeomorphism of the sphere $\mathbb{S}^{2}$ has exactly two fixed points.

An immediate corollary of Theorem 1.1 is that an orientation-preserving, recurrent homeomorphism of the closed disc $D^{2}$ which is the identity on the boundary is the identity on the whole disc. This is the main ingredient used by Oversteegen and Tymchatyn in [16] to show that a recurrent homeomorphism of the Euclidean plane $\mathbb{R}^{2}$ is periodic. However, we do not think that Theorem 1.1 can be proved by a simple rephrasing of the arguments in [16]: one could expect to deduce Theorem 1.1 from the result of Oversteegen and Tymchatyn applying the arguments of section 4 to the non-compact surface $M$ obtained by removing the fixed points of a recurrent homeomorphism of the sphere. The homeomorphism obtained in such a way is clearly recurrent for the induced, restricted metric on $M$ but not necessarily for a hyperbolic metric on $M$. Notice that the analogous statement is false in a simpler case: if one removes one or two fixed points of an irrational rotation of the sphere, the induced homeomorphism on $M$ (the plane or the open annulus) is certainly not recurrent for the euclidean metric, otherwise it would be periodic by [16]. Moreover, our proof relies on a simple property: the fact that a homeomorphism is recurrent is inherited by the induced homeomorphism on prime ends. Up to our knowledge, this elementary idea has not yet been pointed out in the literature.

Even if he does not seem to have published any proof of it, Kerékjártó announced in [13] that a recurrent homeomorphism of a closed, orientable surface of genus $g>1$ is periodic. This result is a corollary of Theorem 1.1 as we shall see in section 4. As noted by Kerékjártó in [12], this implies in particular that a homeomorphism of a compact surface with negative Euler characteristic cannot be topologically transitive provided it has at least one regular point.

The paper is organized as follows: in section 2, we review required material from prime ends' theory. For the proofs in this section, we refer the reader to the works of Epstein [6] or Mather [14]. The proof of Theorem 1.1 is given in section 3 . Section 4 contains several corollaries concerning recurrent homeomorphisms of other surfaces orientable or not.

\section{PRIMe ENDS}

Let $U$ be an open, connected subset of the sphere $\mathbb{S}^{2}$. For a set $A \subset U$ we let $C l(A)$ be its closure in $\mathbb{S}^{2}, C l_{U}(A)$ be its closure in $U, B d(A)$ be its boundary in $\mathbb{S}^{2}$ and $B d_{U}(A)$ be its boundary in $U$.

Definition 2.1. A topological chain is a sequence $\Omega=\left(\Omega_{i}\right)_{i \in \mathbb{N}}$ of open subsets of $U$ such that:

(1) $\Omega_{i}$ and $B d_{U}\left(\Omega_{i}\right)$ are non-empty connected sets;

(2) $C l_{U}\left(\Omega_{i+1}\right) \subset \Omega_{i}$

(3) $C l\left(B d_{U}\left(\Omega_{i}\right)\right) \cap C l\left(B d_{U}\left(\Omega_{j}\right)\right)=\emptyset$ if $i \neq j$;

(4) there exists a point $x \in C l(U)$, the principal point of $\Omega$, such that $x \notin C l\left(B d_{U}\left(\Omega_{i}\right)\right)$, for all $i$ and $B d_{U}\left(\Omega_{i}\right) \rightarrow x$ as $i \rightarrow \infty$.

Two chains $\Omega$ and $\Omega^{\prime}$ are equivalent, denoted by $\Omega \simeq \Omega^{\prime}$, if they divide each other, i.e. if for all $i$ there exists $j$ such that $\Omega_{j}^{\prime} \subset \Omega_{i}$ and $\Omega_{j} \subset \Omega_{i}^{\prime}$. 
The set of all topological chains equivalent to a given chain $\Omega$ is called the prime point defined by $\Omega$, and we write $e=[\Omega]$, or $\Omega \in e$. We denote the set of all the prime points of $U$ by $\hat{U}$.

The set of principal points of chains $\Omega \in e$ is called the principal set of $e$, which we denote by $X(e)$. Let $e$ be a prime point, and let $\Omega$ be any topological chain that defines $e$. The set

$$
Y(e)=\bigcap_{i \in \mathbb{N}} C l\left(\Omega_{i}\right)
$$

depends only on $e$, and is called the impression of $e$. It can be shown $[6,14]$ that $Y(e)$ and $X(e)$ are continua (i.e. non empty, compact, connected sets).

Given $x \in U$, choose any sequence of open discs $\left(D_{i}\right)_{i \in \mathbb{N}}$ such that

$$
C l\left(D_{i+1}\right) \subset D_{i} \subset U \text { and } \bigcap_{i \in \mathbb{N}} C l\left(D_{i}\right)=\{x\} .
$$

The sequence $\left(D_{i}\right)_{i \in \mathbb{N}}$ is a topological chain, and the prime point $\omega(x)$ which it defines depends only on $x$, and not of the particular decreasing sequence $\left(D_{i}\right)_{i \in \mathbb{N}}$. Moreover, we have that

$$
X(\omega(x))=Y(\omega(x))=\{x\} .
$$

This allows us to define a one-to-one map $x \mapsto \omega(x)$ from $U$ into $\hat{U}$, and to consider $U$ as a subset of $\hat{U}$. A prime point which is not in $\omega(U)$ is called a prime end, and it is characterized by the fact that $Y(e) \subset B d(U)$ (see Lemma 2.5 of $[6])$.

A point $x \in B d(U)$ is accessible (from $U$ ) provided that there exists a path $\gamma:[0,1[\rightarrow U$ such that $\gamma(t) \rightarrow x$ as $t \rightarrow 1$. The set of accessible points is dense in $B d(U)$. If $\mathbb{S}^{2} \backslash U$ is the union of a finite number of non-degenerate continua, we have the following lemma.

Lemma 2.2. For each accessible point $x \in B d(U)$, there exists a prime end $e \in \hat{U} \backslash U$ such that $X(e)=\{x\}$

For any open set $V \subset U$, we define

$$
\hat{V}=\left\{[\Omega] \in \hat{U} ; \quad \Omega_{i} \subset V, \quad \text { for some } i\right\} .
$$

With these notations, we get

$$
\hat{V} \cap \hat{W}=\widehat{V \cap W},
$$

and

$$
\hat{V} \cup \hat{W} \subset \widehat{V \cup W} .
$$

Therefore, the family $(\hat{V})_{V \subset U}$ is a basis for a topology $\tau$ on $\hat{U}$. It follows from the definition of this topology that:

(1) if $e=[\Omega]$ then $\left(\hat{\Omega}_{i}\right)_{i \in \mathbb{N}}$ is a basis of neighborhoods of $e$,

(2) $\omega: U \rightarrow \hat{U}$ is a continuous, open map, and $\omega(U)$ is a dense open subset of $\hat{U}$.

The following theorem is a generalization of a theorem due originally to Carathéodory [4] for a simply connected domain. 
Theorem 2.3 ([6], Theorem 6.6). Let $U$ be an open subset of the sphere $\mathbb{S}^{2}$ such that $\mathbb{S}^{2} \backslash U$ is the union of a finite number $p$ of non-degenerate continua. Then, $\hat{U}$, the prime end compactification of $U$ is homeomorphic to a compact surface of genus 0 with $p$ boundary components.

Suppose now that we are given a self-homeomorphism $f$ of the pair $\mathbb{S}^{2}$, such that $f(U)=U$. For each topological chain $\Omega$ with principal point $x, f(\Omega)=\left(f\left(\Omega_{i}\right)\right)_{i \in \mathbb{N}}$ is a topological chain with principal point $f(x)$ and $\Omega^{\prime} \simeq \Omega$ if and only if $f\left(\Omega^{\prime}\right) \simeq f(\Omega)$. Hence $f$ induces a homeomorphism $\hat{f}$ on $\hat{U}$ such that:

(1) for each $V \subset U, \hat{f}(\hat{V})=\widehat{f(V)}$,

(2) for each $e \in \hat{U}, X(\hat{f}(e))=f(X(e))$,

(3) for each $e \in \hat{U}, Y(\hat{f}(e))=f(Y(e))$.

Clearly, if $x \in U$ is a fixed point of $f$ then $\omega(x)$ is a fixed point for $f$. But in general, nothing can be said about fixed prime ends from the existence of fixed points of $f$ lying on $B d(U)$. Moreover, there are simple examples which show that $f$ may have fixed points on $B d(U)$ although $\hat{f}$ has no fixed point at all. However, if $e$ is a fixed prime end and $X(e)=\{x\}$ then $f(x)=x$ since

$$
\{f(x)\}=X(\hat{f}(e))=X(e)=\{x\} .
$$

The following result, which we state without proof, is a consequence of Lemma 2.2 and of the density of accessible points in $B d(U)$.

Corollary 2.4. Let $f$ be a homeomorphism of $\mathbb{S}^{2}$ and $U$ an invariant open set such that $\mathbb{S}^{2} \backslash U$ is the union of a finite number of non-degenerate continua. If $\hat{f}=\hat{I d}$ on $\hat{U} \backslash \omega(U)$, then $f=I d$ on $B d(U)$.

The following lemma is new. The fact that a homeomorphism is recurrent is inherited by the induced homeomorphism on prime ends seems not to have been pointed out in the literature, before.

Lemma 2.5. Let $f$ be a recurrent homeomorphism of the sphere $\mathbb{S}^{2}$ and $U$ an invariant open set, such that $\mathbb{S}^{2} \backslash U$ is the union of a finite number of non-degenerate continua. Then, the homeomorphism $\hat{f}$ induced by $f$ on $\hat{U}$ is recurrent.

Before giving the proof of this result, we recall an elementary lemma of topology widely used in the theory of prime ends [14].

Lemma 2.6. Let $A$ and $B$ be open subsets of a topological space $X$. Suppose that $X, A, B, B d_{X}(A)$ and $B d_{X}(B)$ are non-empty connected sets, and that $B d_{X}(A) \cap B d_{X}(B)=\emptyset$. Then exactly one of the following situations holds:

(1) $X=A \cup B$,

(2) $C l_{X}(A) \cap C l_{X}(B)=\emptyset$,

(3) $C l_{X}(A) \subset B$,

(4) $C l_{X}(B) \subset A$.

Proof of Lemma 2.5. We fix a metric on the compact surface of finite type $\hat{U}$, and to avoid any confusion with the metric $d$ on $U$, we denote it by $\alpha$. Choose a sequence $\left(n_{k}\right)$ such that

$$
d\left(f^{n_{k}}, I d\right) \rightarrow 0
$$


on $C l(U)$. Let $\varepsilon>0, e \in \hat{U}$ and $B_{\alpha}(e, \varepsilon)$ be the ball of radius $\varepsilon$ around $e$. For any chain $\Omega=\left(\Omega_{i}\right)_{i \in \mathbb{N}}$ that defines $e$, we have $\hat{\Omega}_{i} \subset B_{\alpha}(e, \varepsilon)$, for large enough $i$. Hence we can assume that $\hat{\Omega}_{1} \subset B_{\alpha}(e, \varepsilon)$.

For $k$ large enough, say for $k$ greater than some integer $k(e, \varepsilon)$, the hypothesis of Lemma 2.6 applies to the sets $X=U, A=\Omega_{1}$ and $B=f^{n_{k}}\left(\Omega_{2}\right)$. The only possibility among the four alternative is the fourth one and so

$$
f^{n_{k}}\left(\Omega_{2}\right) \subset \Omega_{1} \text { for all } k \geq k(e, \varepsilon) .
$$

Therefore, we have found a neighbourhood $\mathcal{V}_{e}=\widehat{\Omega}_{2}$ of $e$, and an integer $k(e, \varepsilon)$ so that

$$
\mathcal{V}_{e} \subset B_{\alpha}(e, \varepsilon) \text { and } \hat{f}^{n_{k}}\left(\mathcal{V}_{e}\right) \subset B_{\alpha}(e, \varepsilon),
$$

for all $k \geq k(e, \varepsilon)$. Since $\hat{U}$ is compact, only a finite number of $\mathcal{V}_{e}$, say $\mathcal{V}_{e_{1}}, \mathcal{V}_{e_{2}}, \cdots, \mathcal{V}_{e_{r}}$, are necessary to cover $\hat{U}$, and hence, for

$$
k \geq \max \left\{k\left(e_{1}, \varepsilon\right), k\left(e_{2}, \varepsilon\right), \ldots, k\left(e_{r}, \varepsilon\right)\right\},
$$

we have $\alpha\left(f^{n_{k}}, I d\right) \leq 2 \varepsilon$. In other words, $\hat{f}$ is recurrent.

\section{Proof OF THE MAIN PROPOSITION}

The proof of Theorem 1.1 relies heavily on the construction, for every fixed point of $f$, of arbitrarily small, invariant, non degenerate continua containing the fixed point: the so-called Birkhoff construction.

Lemma 3.1 (Birkhoff Construction). Let $f$ be a recurrent homeomorphism of the sphere $\mathbb{S}^{2}, x_{0}$ a fixed point of $f$ and $D$, a topological disc, containing $x_{0}$, and bounded by a simple closed curve $c$. There exists a simply-connected continuum $K$, such that:

(1) $x_{0} \in K \subset C l(D)$,

(2) $f(K)=K$,

(3) $K \cap c \neq \emptyset$.

Proof. We define inductively a decreasing sequence of Jordan domains, $D_{n}$, by the following properties

(1) $D_{0}=D$,

(2) $D_{n+1}$ is the connected component of $x_{0}$ in $f\left(D_{n}\right) \cap D_{0}$.

That each $D_{n}$ is a Jordan domain can be established by induction on $n$ : if $f\left(D_{n}\right) \subset D_{0}$ or $D_{0} \subset f\left(D_{n}\right)$, there is nothing to prove. Otherwise, the two simple closed curves $B d\left(D_{0}\right)$ and $f\left(B d\left(D_{n}\right)\right)$ have at least two common points and $D_{n+1}$ is a Jordan domain (cf. Theorem 16.3 of [15]).

As the intersection of decreasing discs, the set

$$
K=\bigcap_{n \in \mathbb{N}} C l\left(D_{n}\right)
$$

is a simply-connected continuum. It is contained in $C l(D)$ and contains $x_{0}$. Moreover $K \subset f(K)$ and since $f$ is recurrent this implies $f(K)=K$.

It remains to show that $K \cap c \neq \emptyset$, the proof of which is a consequence of the intersecting curve property, verified by a recurrent homeomorphism. 
Suppose on the contrary that $K \cap c=\emptyset$. In that case, we can find an integer $n_{0} \geq 0$ such that

$$
C l\left(D_{n_{0}+1}\right) \cap c=\emptyset
$$

and hence

$$
B d\left(D_{n_{0}+1}\right) \subset B d\left(f\left(D_{n_{0}}\right)\right),
$$

which leads to

$$
D_{n_{0}+1}=f\left(D_{n_{0}}\right) .
$$

But then, we have

$$
B d\left(D_{n_{0}+2}\right) \subset B d\left(f\left(D_{n_{0}+1}\right)\right)=B d\left(f^{2}\left(D_{n_{0}}\right)\right),
$$

and hence

$$
D_{n_{0}+2}=f^{2}\left(D_{n_{0}}\right)
$$

Iterating the process inductively, we obtain that

$$
D_{n_{0}+i}=f^{i}\left(D_{n_{0}}\right)
$$

for all $i \geq 0$, and hence that

$$
K=\bigcap_{i \in \mathbb{N}} C l\left(f^{i}\left(D_{n_{0}}\right)\right) .
$$

But then $K \cap c_{n_{0}} \neq \emptyset$, otherwise, we could find $k \in \mathbb{N}$ such that

$$
C l\left(f^{k}\left(D_{n_{0}}\right)\right) \cap c_{n_{0}}=\emptyset,
$$

which is not possible since $f$ is recurrent. Moreover, since

$$
c_{n_{0}} \subset c \cup f(c) \cup \cdots \cup f^{n_{0}}(c),
$$

and $f(K)=K$, the existence of a point in $K \cap c_{n_{0}}$ would lead to the existence of a point in $K \cap c$, which gives a contradiction and completes the proof.

Proposition 3.2. Let $f$ be a recurrent, orientation-preserving homeomorphism of the sphere $\mathbb{S}^{2}$. If $f$ has three fixed point, then Fix $(f)$ is connected.

Proof. Suppose on the contrary that Fix $(f)$ is not connected. Then, we can write

$$
F i x(f)=X \cup Y,
$$

where $X$ and $Y$ are two disjoint, non-empty closed sets. Let $x \in X$ and $y \in Y$. We can find a Jordan curve $c$, in the complement of Fix $(f)=X \cup Y$, which separates $x$ from $y$ (see [15] or [17]). Let $D_{x}$ (resp. $D_{y}$ ) be the component of $\mathbb{S}^{2} \backslash c$ which contains $x$ (resp. y). By hypothesis, $f$ has a third fixed point, and hence, at least one component of $\mathbb{S}^{2} \backslash c$, say $D_{x}$, contains two fixed points $x$ and $z$. Let $J \subset D_{x}$ be a simple closed curve which separates $x$ and $z$ in $D_{x}$, and let $\Delta_{x}$ be the component of $\mathbb{S}^{2} \backslash J$ that contains $x$. Using the construction of Lemma 3.1, first for $x$ and $\Delta_{x}$ and then for $y$ and $D_{y}$, we obtain two disjoint, invariant, non-degenerate, simply connected continua $K_{x}$ and $K_{y}$ such that

$$
\emptyset \neq K_{y} \cap c \subset B d\left(K_{y}\right) \cap c \subset \mathbb{S}^{2} \backslash F i x(f)
$$

The set $U=\mathbb{S}^{2} \backslash\left(K_{x} \cup K_{y}\right)$ is an open topological annulus which contains $z$ and which is invariant under $f$. According to Theorem 2.3, $\hat{U}$ is homeomorphic to a closed annulus and due to Lemma 2.5, the induce homeomorphism 
$\hat{f}$ is recurrent. Moreover, $\hat{f}$ is orientation-preserving, boundary-preserving and has a fixed point $\omega(z)$.

In general, a lift $F$ of $\hat{f}$ to $\mathbb{R} \times[0,1]$ needs not be recurrent. However if we choose a lift $F$ which has a fixed point, it is recurrent. Therefore, on each line $\mathbb{R} \times\{i\},(i=0,1), F$ is the identity. Hence $\hat{f}$ itself is the identity on $\hat{U} \backslash U$ and according to Corollary 2.4, $f$ is the identity on $B d(U)$. But $B d(U)$ meets $c$ which lies in $\mathbb{S}^{2} \backslash F i x(f)$. This gives a contradiction and completes the proof.

Proof of Theorem 1.1. Let $f$ be an orientation-preserving homeomorphism of $\mathbb{S}^{2}$. According to Brouwer's fixed point theorem for the sphere, $f$ has at least one fixed point $x$. Moreover, if $f$ is recurrent then $f$ induces an orientation-preserving homeomorphism of

$$
\mathbb{S}^{2} \backslash\{x\} \simeq \mathbb{R}^{2}
$$

for which each point is recurrent. According to Brouwer's lemma on translation arcs [2,9], which asserts that all points of an orientation-preserving, fixed point free homeomorphism of the plane are wandering, $f$ has necessarily a second fixed point $y$.

Suppose that $f$ has a third fixed point. According to Proposition 3.2, Fix $(f)$ is connected. Hence, each component of $\mathbb{S}^{2} \backslash F i x(f)$ is a simply connected domain of $\mathbb{S}^{2}$ homeomorphic to the plane $\mathbb{R}^{2}$.

According to a result of Brown and Kister [3], an orientation-preserving homeomorphism of the sphere leaves invariant each component of the complement of its fixed point set.

Hence, if Fix $(f) \neq \mathbb{S}^{2}$ there exists a component $U \simeq \mathbb{R}^{2}$ of $\mathbb{S}^{2} \backslash F i x(f)$ such that $f(U)=U$ and $F i x\left(f_{U}\right)=\emptyset$. But each point of $U$ is recurrent under $f$, this is once again a contradiction with Brouwer's lemma on translation arcs. This completes the proof of Theorem 1.1.

Corollary 3.3. A recurrent orientation-reversing homeomorphism of the sphere which has a fixed point is an involution.

Proof. Let $f$ be a recurrent, orientation-reversing homeomorphism of the sphere. Suppose that $f$ has a fixed point $x$ but $f^{2} \neq I d$. According to Theorem 1.1, $f^{2}$ has only two fixed points $x$ and $y$ and since $f(x)=x$, we must have

$$
F i x(f)=\{x, y\} .
$$

The Birkhoff's construction may be applied to obtain two distinct, $f$-invariant, simply connected continua $K_{x}$ and $K_{y}$. As described in sections 2 and $3, f$ induces a recurrent, orientation-reversing homeomorphism $\hat{f}$ on the prime end compactification of $\mathbb{S}^{2} \backslash\left(K_{x} \cup K_{y}\right)$ which is a closed annulus.

Since $\hat{f}$ preserves the boundary component of this annulus and reverse the orientation, it must have a fixed point according to the Lefschetz trace formula. Hence, $\hat{f}^{2}$ must be the identity on the boundary of the annulus. But this implies that $f^{2}=I d$ on $B d\left(K_{x} \cup K_{y}\right)$ which leads to a contradiction as in the proof of Theorem 1.1.

Corollary 3.4. A non trivial, recurrent, orientation-preserving homeomorphism of the disc has a unique fixed point. A recurrent, orientation-reversing homeomorphism of the disc is an involution. 
Proof. Taking the double of a recurrent, orientation-preserving homeomorphism of the disc, we obtain at once the first part of Corollary 3.4 as a consequence of Theorem 1.1. Let us now consider a recurrent, orientationreversing homeomorphism $f$ of the disc. As an orientation-reversing homeomorphism of the circle, the restriction of $f$ to the boundary of the disc has a fixed point and hence $f^{2}=I d$ on the boundary of the disc. By previous considerations, this leads to $f^{2}=I d$ and completes the proof.

\section{RECURRENT homeOMORPHisms of SURFACES}

Let $M^{2}$ be a closed orientable surface of genus $g>1$ and $\pi: \tilde{M}^{2} \rightarrow M^{2}$ the universal cover of $M^{2}$. We can identify $\tilde{M}^{2}$ either to the euclidean plane $\mathbb{R}^{2}$ or to the Poincaré disc $\mathbb{D}$ in such a way that $M^{2}$ is homeomorphic to the quotient of $M^{2}$ by a discrete subgroup $\Gamma$ of euclidean translations or hyperbolic isometries according to whether $M^{2}$ is $\mathbb{R}^{2}$ or $\mathbb{D}$. The metric we shall use on $M^{2}$ is the quotient metric on $M^{2} / \Gamma$ defined by

$$
d(\pi(x), \pi(y))=\inf _{g, h \in \Gamma} \tilde{d}(g . x, h . y),
$$

where $\tilde{d}$ is the natural metric on $\tilde{M}^{2}$.

There is another metric on $\tilde{M}^{2}$ that we shall use in the following, namely the spherical metric. The Alexandroff compactification $\tilde{M}^{2} \cup\{\infty\}$, is homeomorphic to the sphere $\mathbb{S}^{2}$. The standard metric of $\mathbb{S}^{2}$ induces a metric $\partial$ on $\tilde{M}^{2}$ that we call the spherical metric. These two metrics $\tilde{d}$ and $\partial$ are not uniformly equivalent on $\tilde{M}^{2}$ but $I d:\left(\tilde{M}^{2}, \tilde{d}\right) \rightarrow\left(\tilde{M}^{2}, \partial\right)$ is uniformly continuous.

Lemma 4.1. Let $f$ be an orientation-preserving recurrent homeomorphism of a closed orientable surface $M^{2}$ of genus $g>1$. If $f$ has a fixed point and acts trivially on $\pi_{1}\left(M^{2}\right)$ then $f=I d$.

Proof. A lift of a recurrent homeomorphism of $M^{2}$ needs not be recurrent in general. However, a lift $\tilde{f}$ which has a fixed point is recurrent for the metric $d$. The fact that $I d:\left(\tilde{M}^{2}, \tilde{d}\right) \rightarrow\left(\tilde{M}^{2}, \partial\right)$ is uniformly continuous is enough to ensure that the extension of $\tilde{f}$ to $\mathbb{S}^{2}$ (letting $\tilde{f}(\infty)=\infty$ ) is recurrent for the metric $\partial$. Moreover if $f$ acts trivially on $\pi_{1}\left(M^{2}\right), \tilde{f}$ has infinitely many fixed points since it commutes with all covering translations and hence $\tilde{f}=I d$ according to Theorem 1.1.

Corollary 4.2. A recurrent homeomorphism of a compact surface with negative Euler characteristic is periodic.

Proof. We first note that if the boundary of $M^{2}$ is not empty, the natural extension of $f$ to the double $D M^{2}$ of $M^{2}$ is still recurrent and since $\chi\left(D M^{2}\right)=2 \chi\left(M^{2}\right)$, we are reduced to prove Corollary 4.2 for closed surfaces. Moreover, by passing to the orientation covering of $M^{2}$ and considering $f^{2}$ instead of $f$ if necessary, we may assume that $M^{2}$ is orientable with genus $g>1$ and that $f$ is orientation-preserving. Recall then that every homeomorphism of $M^{2}$ which is close enough to the identity must be homotopic to the identity. Hence, since $f$ is recurrent we can find a positive integer $n$ such that $f^{n}$ is homotopic to the identity. The Lefschetz number 
of $g=f^{n}$ is thus $L(g)=\chi\left(M^{2}\right)<0$ and $g$ has a fixed point. According to Lemma 4.1, we must have then $g=I d$ which completes the proof.

Remark 4.3. We emphasize on the fact that Corollary 4.2 is false for surfaces with non negative Euler characteristic. Indeed, the example of [7] can be modified to exhibit non-regular, recurrent homeomorphisms on the projective plane, the torus, the Klein bottle, the annulus, the Möbius strip or the disc. However, for surfaces with $\chi\left(M^{2}\right)=0$, namely the torus, the Klein bottle, the annulus and the Möbius strip, the proof of Corollary 4.2 shows that the existence of a periodic point for a recurrent homeomorphism implies that this homeomorphism is periodic itself.

Corollary 4.4. A non-trivial, recurrent homeomorphism of the projective plane $\mathbb{P}^{2}$ admit a unique fixed point or is an involution.

Proof. We shall use as model for the real projective plane $\mathbb{P}^{2}$, the quotient of the sphere $\mathbb{S}^{2}$ by the involution $\theta: x \mapsto-x$. Let $f$ be a recurrent homeomorphism of the projective plane $\mathbb{P}^{2} . f$ has two lifts on $\mathbb{S}^{2}$ which commute with $\theta$. One of them $\tilde{f}_{+}$is orientation preserving and the other $\tilde{f}_{-}=\theta \circ \tilde{f}_{+}$is orientation-reversing. $\tilde{f}_{+}$has a fixed point and is therefore recurrent as we have already noticed. Therefore, if $f$ is not trivial, $\tilde{f}_{+}$has exactly two fixed point $x$ and $\theta(x)$ which project down to one fixed point of $f$. Hence, the existence of a second fixed point of $f$ implies that $\tilde{f}_{-}$has a fixed point and is therefore recurrent. It must satisfy $\tilde{f}_{-}^{2}=I d$ according to Corollary 3.3. This shows that $f$ must be an involution and completes the proof.

Remark 4.5. Recall that a regular point for a homeomorphism $f$ on a compact metric space $(X, d)$ is a point at which the family of all the iterates $\left\{f^{n} ; n \in \mathbb{Z}\right\}$ is equicontinuous. As noted in the introduction, Corollary 4.2 is the key argument to show that a homeomorphism of a compact surface with negative Euler characteristic which has a regular point cannot be topologically transitive and hence cannot be ergodic with respect to an invariant measure $\mu$ such that $\mu(U)>0$ for all open sets $U$. Indeed, the orbit of a regular point cannot be a dense set, otherwise the homeomorphism is recurrent, and hence periodic, which gives a contradiction. But the presence of a regular point with a non dense orbit implies existence of proper, non empty invariant open sets.

\section{REFERENCES}

[1] C. Bonatti and B. Kolev. Surface homeomorphisms with zero-dimensional singular set. Topology Appl., 90(1-3):69-95, 1998.

[2] M. Brown. A new proof of Brouwer's lemma on translation arcs. Houston J. Math., 10(1):35-41, 1984.

[3] M. Brown and J. M. Kister. Invariance of complementary domains of a fixed point set. Proc. Amer. Math. Soc., 91(3):503-504, 1984.

[4] C. Carathéodory. Über die Begrenzung einfach zusammenhängender Gebiete. Math. Ann., 73(3):323-370, 1913.

[5] A. Constantin and B. Kolev. The theorem of Kerékjártó on periodic homeomorphisms of the disc and the sphere. Enseign. Math. (2), 40(3-4):193-204, 1994.

[6] D. B. A. Epstein. Prime ends. Proc. London Math. Soc. (3), 42(3):385-414, 1981. 
[7] R. J. Fokkink and L. G. Oversteegen. A recurrent nonrotational homeomorphism on the annulus. Trans. Amer. Math. Soc., 333(2):865-875, 1992.

[8] W. H. Gottschalk. Minimal sets: an introduction to topological dynamics. Bull. Amer. Math. Soc., 64:336-351, 1958.

[9] L. Guillou. Théorème de translation plane de Brouwer et généralisations du théorème de Poincaré-Birkhoff. Topology, 33(2):331-351, 1994.

[10] B. von Kérékjartó. Über die periodischen Transformationen der Kreisscheibe und der Kugelfläche. Math. Ann., 80:36-38, 1919-1920.

[11] B. von Kérékjartó. Sur le caractere topologique des representations conformes. $C$. $R$. Acad. Sci., Paris, 198:317-320, 1934.

[12] B. von Kérékjartó. Stabilité permanente et l'hypothese ergodique. C. R. Acad. Sci., Paris, 201:123-124, 1935.

[13] B. von Kérékjartó. Sur la structure des transformations topologiques des surfaces en elles-mêmes. Enseign. Math., 35:297-316, 1936.

[14] J. N. Mather. Topological proofs of some purely topological consequences of Carathéodory's theory of prime ends. In Selected studies: physics-astrophysics, mathematics, history of science, pages 225-255. North-Holland, Amsterdam, 1982.

[15] M. H. A. Newman. Elements of the topology of plane sets of points. Dover Publications Inc., New York, 1992.

[16] Lex G. Oversteegen and E. D. Tymchatyn. Recurrent homeomorphisms on $\mathbf{R}^{2}$ are periodic. Proc. Amer. Math. Soc., 110(4):1083-1088, 1990.

[17] G.T. Whyburn. Topological analysis. Second, revised edition. Princeton Mathematical Series, No. 23. Princeton University Press, Princeton, N.J., 1964.

CMi, 39 rue F. Joliot-Curie, 13453 Marseille Cedex 13, France

E-mail address: kolev@cmi.univ-mrs.fr 